\title{
The Glass Transition Within the Thermodynamics of Irreversible Processes
}

\author{
H. Baur \\ Sonnenwendstr. 41, D-67098 Bad Dürkheim \\ Z. Naturforsch. 53a, 157-170 (1998); received December 29, 1997
}

\begin{abstract}
The glass transition caused by a finite cooling rate is a continuous non-linear dissipative process whose description requires a clear distinction between equilibrium and non-equilibrium quantities. The socalled Davies or Prigogine-Defay relations (in form of an equation as well as in form of an inequality) are not relevant in such a process. The determining quantities of the glass transition are - from a macroscopic phenomenological point of view - the fluidity of the melt and the partial free enthalpy of the microscopic vacancies in the melt. All of the characteristics of the dynamics of the glass transition are essentially due to these two quantities.
\end{abstract}

\section{Introduction}

Simon [1] concluded from the fact that a finite entropic difference is retained between the vitreous state and the crystalline state of a pure substance in the limit $T \rightarrow 0$ ( $T$ : absolute temperature) that one is not dealing with an internal equilibrium state in the case of a glass but rather with a "frozen" non-equilibrium state. Within the definitions of the thermodynamics of irreversible processes, the vitreous state, therefore, has to be classified as an arrested equilibrium state [2, 3].

In the following, we will explicitly assign a (macroscopic) internal variable $\zeta$ to the molecular internal degree of freedom frozen (arrested) in the vitreous state. When cooling a liquid at constant pressure $p$ at a constant and not too high rate $\dot{T}=\beta<0$, one schematically obtains the following picture, for example, for the volume $V$ (Figure 1): To begin with, one should take into account that every process which occurs at a finite rate $\dot{T} \neq 0$ is an irreversible process connected with a finite entropy production. In this case, the volume follows a nonequilibrium curve $V(T, \zeta)$ in which the temperature $T$ and the internal variable $\zeta$ vary independently of each other. However, if one cools, proceeding from a temperature $T_{0}$ at a not too high rate $\beta=$ const., a volume is measured at the beginning of the process which practically follows the curve $V\left(T, \zeta_{\mathrm{e}}(T)\right)$ of the internal equilibrium of the liquid. The development of this curve is uniquely determined by the temperature. At a temperature $T_{\mathrm{F}}<T_{0}$, however the volume curve starts to considerably deviate from the equilibrium curve. Below $T_{\mathrm{F}}$, the internal variable $\zeta$

Reprint requests to Priv. Doz. Dr. H. Baur. clearly deviates from its equilibrium value $\zeta_{\mathrm{e}}(T)$, and the volume curve $V(T, \zeta)$ is no longer uniquely determined by the temperature. At a temperature $T_{\mathrm{g}}^{\prime}$, the internal molecular degree of freedom then freezes with $\dot{\zeta} \approx 0$. Subsequently, the volume follows the volume curve $V(T$, $\zeta=$ const.) of the arrested equilibrium, whose development depends solely on the temperature, but whose level, on the other hand, is determined by the non-equilibrium value $\zeta\left(T_{\mathrm{g}}^{\prime}\right)$ of the internal variable frozen in at $T_{\mathrm{g}}^{\prime}$. The glass transition region, i.e., the position of the temperature interval $\left\langle T_{\mathrm{g}}^{\prime}, T_{\mathrm{F}}\right\rangle$, as well as the non-equilibrium value $\zeta$, which freezes in at $T_{\mathrm{g}}^{\prime}$, depends on the cooling rate $\dot{T}=\beta<0$. According to the statements pointed out

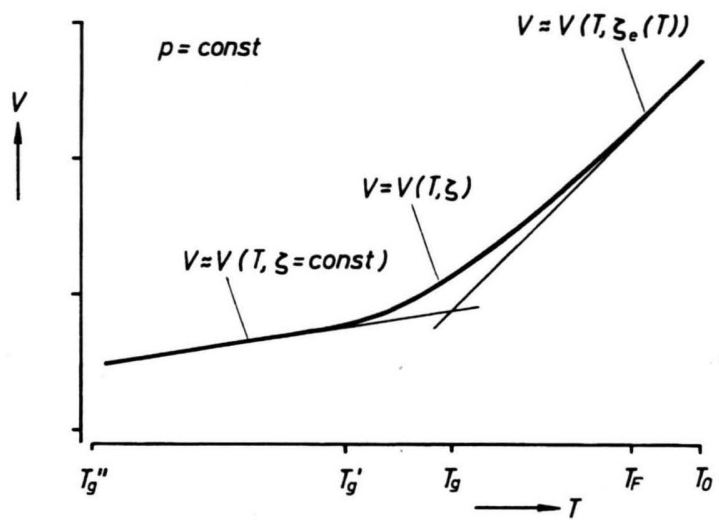

Fig. 1. Volume $V$ as a function of the temperature $T$ when cooling a non-crystallizing melt with a not too high constant cooling rate $T$ under constant pressure $p$. $\zeta$ : internal variable; $\zeta_{\mathrm{e}}(T)$ : its equilibrium value; $T_{0}$ : initial temperature; $T_{\mathrm{F}}$ : temperature at which first deviations from the internal equilibrium become noticeable; $T_{\mathrm{g}}$ : so-called glass temperature; $T_{\mathrm{g}}^{\prime}$ : freezing temperature; $T_{\mathrm{g}}^{\prime \prime}:$ Vogel temperature (see Sect. 5). 
in Sect. 5, however, the internal degree of freedom does not freeze completely at $T_{\mathrm{g}}^{\prime}$. Very small changes in $\zeta$ are still found below $T_{\mathrm{g}}^{\prime}$. A complete arrest of the internal degree of freedom with $\dot{\zeta}=0$ is only observed normally at a considerably lower temperature $T_{\mathrm{g}}^{\prime \prime}$. The position of $T_{\mathrm{g}}^{\prime}$ decreases with the cooling rate $\beta$. In the limit $\beta \rightarrow 0$, i.e., in the case of a fictitious, pure equilibrium process, $T_{\mathrm{g}}^{\prime}$ finally reaches the value $T_{\mathrm{g}}^{\prime \prime}$.

If one proceeds from the linearized mechanical equations of state, one obtains at constant pressure $p$ for the internal equilibrium the straight line

$$
V=V_{\mathrm{g}}\left[1-\alpha_{\mathrm{e}}\left(T-T_{\mathrm{g}}\right)\right], T \geq T_{\mathrm{g}}
$$

and for the arrested equilibrium the straight line

$$
V=V_{\mathrm{g}}\left[1-\alpha_{\zeta_{\mathrm{e}}}\left(T-T_{\mathrm{g}}\right)\right], T \leq T_{\mathrm{g}}
$$

[ $\alpha_{\mathrm{e}}$ and $\alpha_{\zeta_{\mathrm{e}}}$ are the coefficients of thermal expansion of the internal and the arrested equilibria (see Sect. 2)]. The two intersect at $T_{\mathrm{g}}$, the so-called glass temperature. In this approximation (the so-called "simple freezing-in model"; Rehage [3]), the glass transition region is reduced to a single temperature $T_{\mathrm{g}}$. The glass transition becomes a discontinuous process. The value $\zeta_{\mathrm{g}}$ of the internal variable, which freezes at $T_{\mathrm{g}}$, is necessarily the equilibrium value $\zeta_{\mathrm{g}}=\zeta_{\mathrm{e}}\left(T_{\mathrm{g}}\right)$ in this model. The coefficient of thermal expansion suffers a discontinuous jump at $T_{\mathrm{g}}$ :

$$
\Delta^{\mathrm{e}} \alpha=\alpha_{\mathrm{e}}-\alpha_{\zeta \mathrm{e}}
$$

Physically, $T_{\mathrm{g}}$ and $\zeta_{\mathrm{e}}\left(T_{\mathrm{g}}\right)$ are, of course, purely fictitious quantities. Nevertheless, $T_{\mathrm{g}}$ is well-defined. The temperatures $T_{\mathrm{F}}$ and $T_{\mathrm{g}}^{\prime}$, which are characteristic for the real glass transition, on the other hand, can only be determined rather vaguely. This is due to the fact that, as already mentioned, the volume curve measured at a finite cooling rate is a non-equilibrium curve which develops continuously in the whole range $\left\langle T_{\mathrm{g}}^{\prime \prime}, T_{0}\right\rangle$.

\section{The Fundamental Relations of the Thermo- dynamics of Irreversible Processes*}

In the following, we will consider a homogeneous, fluid single-component system with constant mass. According to the assumptions of the thermodynamics of irreversible processes, the Gibbs formalism of equilibrium thermodynamics also holds in non-equilibrium for such systems. The Gibbs potentials, however, must be complemented by the so-called internal variables, which

\footnotetext{
* For example, compare [4-6] or [26].
}

represent the additional degrees of freedom in non-equilibrium. In the following, we will only consider a single internal variable $\zeta$. In the G-representation, Gibbs' fundamental equation of the system is then

$$
g=g(T, p, \zeta)=h(T, p, \zeta)-T s(T, p, \zeta) .
$$

It connects the specific free enthalpy $g$ with the mutually independent variables $T, p, \zeta$ of the system.

$$
s \equiv-\left(\frac{\partial g}{\partial T}\right)_{p, \zeta} \text { and } h \equiv g-T\left(\frac{\partial g}{\partial T}\right)_{p, \zeta}
$$

are the specific entropy and the specific enthalpy of the system.

In non-equilibrium, the independent variables $T(t)$, $p(t), \zeta(t)$ are explicit functions of time $t$. If the temperature and the pressure of the system are also identical with the temperature and the pressure of the surroundings of the system in non-equilibrium, the changes with time $\dot{T} \equiv \mathrm{d} T / \mathrm{d} t$ and $\dot{p} \equiv \mathrm{d} p / \mathrm{d} t$ are determined by the manner of external perturbation of the system. One can assume a simple dynamic law in the form

$$
\dot{\zeta}=L a, \quad L \geq 0
$$

for the change of the internal variable with time. $a(T, p$, $\zeta$ ) is the so-called affinity, which can be thought of as a driving force for the internal variable. The affinity is given by

$$
a \equiv-\left(\frac{\partial g}{\partial \zeta}\right)_{T, p}=T \sigma_{T p}-\eta_{T p},
$$

whereby

$$
\sigma_{T p} \equiv\left(\frac{\partial s}{\partial \zeta}\right)_{T, p} \text { and } \quad \eta_{T p} \equiv\left(\frac{\partial h}{\partial \zeta}\right)_{T, p}
$$

denote the partial specific entropy and the partial specific enthalpy relative to the relevant internal degree of freedom.

Thermodynamic quantities which refer to an internal equilibrium state are indicated with "e". In the internal equilibrium, the affinity goes to zero. Thus, an internal equilibrium state is characterized by

$$
a_{\mathrm{e}}=0 \text { or } T \sigma_{T p}^{\mathrm{e}}=\eta_{T p}^{\mathrm{e}} .
$$

This equation fixes one of the variables $T, p, \zeta$ as a function of the other, for example

$$
\zeta=\zeta_{\mathrm{e}}(T, p) \text {. }
$$

Although the internal variable remains variable in internal equilibrium, it is no longer an independent variable. 
In addition to $(6 \mathrm{a})$,

$$
(\mathrm{d} a)_{\mathrm{e}}=0
$$

must be fulfilled in the internal equilibrium. For the change in the affinity with the independent variables, one gets:

$$
\mathrm{d} a=\left(\frac{\partial a}{\partial T}\right)_{p, \zeta} \mathrm{d} T+\left(\frac{\partial a}{\partial p}\right)_{T, \zeta} \mathrm{d} p+\left(\frac{\partial a}{\partial \zeta}\right)_{T, p} \mathrm{~d} \zeta .
$$

According to (2) and (4), one obtains in the internal equilibrium:

$$
\begin{aligned}
& \left(\frac{\partial a}{\partial T}\right)_{p, \zeta}^{\mathrm{e}}=-\left(\frac{\partial^{2} g}{\partial T \partial \zeta}\right)_{\mathrm{e}}=-\left(\frac{\partial^{2} g}{\partial \zeta \partial T}\right)_{\mathrm{e}}=\sigma_{T p}^{\mathrm{e}}, \\
& \left(\frac{\partial a}{\partial \zeta}\right)_{T, p}^{\mathrm{e}}=-\left(\frac{\partial^{2} g}{\partial \zeta^{2}}\right)_{T, p}^{\mathrm{e}} \equiv-\gamma_{T p}^{\mathrm{e}} .
\end{aligned}
$$

Hence, according to (6b), the change of the internal variable with the temperature at constant pressure in the internal equilibrium is given by

$$
\left(\frac{\mathrm{d} \zeta}{\mathrm{d} T}\right)_{p}^{\mathrm{e}}=\frac{\sigma_{T p}^{\mathrm{e}}}{\gamma_{T p}^{\mathrm{e}}}
$$

In addition, we have in a stable or metastable internal equilibrium

$$
\gamma_{T p}^{\mathrm{e}}>0 .
$$

For the change in the entropy with respect to the independent variables, one obtains

$$
\mathrm{d} s=\left(\frac{\partial s}{\partial T}\right)_{p, \zeta} \mathrm{d} T+\left(\frac{\partial s}{\partial p}\right)_{T, \zeta} \mathrm{d} p+\left(\frac{\partial s}{\partial \zeta}\right)_{T, p} \mathrm{~d} \zeta .
$$

For the specific heat capacity at constant pressure, this leads to

$$
c_{p} \equiv T\left(\frac{\mathrm{d} s}{\mathrm{~d} T}\right)_{p}=T\left(\frac{\partial s}{\partial T}\right)_{p, \zeta}+T \sigma_{T p}\left(\frac{\mathrm{d} \zeta}{\mathrm{d} T}\right)_{p} .
$$

Here,

$$
c_{p, \zeta} \equiv T\left(\frac{\partial s}{\partial T}\right)_{p, \zeta}
$$

is the specific heat capacity of the arrested equilibrium ( $\zeta=$ const.). This heat capacity is measured when the change in temperature occurs so rapidly that the internal degree of freedom is not capable of following this change
( $\dot{\zeta} \ll \dot{T}$ ), or if the internal degree of freedom is frozen ( $\dot{\zeta}=0$, because of $L \rightarrow 0$; see below). In the internal equilibrium, one measures, according to (8), the heat capacity

$$
c_{p}^{\mathrm{e}}=c_{p, \zeta_{\mathrm{e}}}+\Delta^{\mathrm{e}} c_{p}
$$

with

$$
\Delta^{\mathrm{e}} c_{p}=\frac{T\left(\sigma_{T p}^{\mathrm{e}}\right)^{2}}{\gamma_{T p}^{\mathrm{e}}} \geq 0 .
$$

$\Delta^{\mathrm{e}} c_{p}$ is the contribution of the internal degree of freedom to the heat capacity in the internal equilibrium. Outside the equilibrium, i.e. in non-equilibrium, $T$ and $\zeta$ are mutually independent but time-dependent variables. With $p=$ const., one must then set

$$
\left(\frac{\mathrm{d} \zeta}{\mathrm{d} T}\right)_{p}=\dot{\zeta} / \dot{T}
$$

Under conditions of non-equilibrium, one measures, according to (11), the heat capacity

$$
c_{p}=c_{p, \zeta}+\Delta c_{p}
$$

with

$$
\Delta c_{p}=T \sigma_{T p} \frac{\dot{\zeta}}{\dot{T}} .
$$

$\Delta c_{p}$ is the contribution of the internal degree of freedom to the heat capacity under conditions of non-equilibrium.

Corresponding expressions can be derived for the coefficient of thermal expansion $\alpha$ and for the isothermal compressibility $\kappa_{T}$. One obtains as the coefficient of thermal expansion:

$$
\begin{aligned}
\alpha & \equiv \frac{1}{v}\left(\frac{\mathrm{d} v}{\mathrm{~d} T}\right)_{p} \\
& =\frac{1}{v}\left(\frac{\partial v}{\partial T}\right)_{p, \zeta}+\frac{1}{v} \varphi_{T p}\left(\frac{\mathrm{d} \zeta}{\mathrm{d} T}\right)_{p},
\end{aligned}
$$

and as the compressibility:

$$
\begin{aligned}
\kappa_{T} & \equiv-\frac{1}{v}\left(\frac{\mathrm{d} v}{\mathrm{~d} p}\right)_{T} \\
& =-\frac{1}{v}\left(\frac{\partial v}{\partial p}\right)_{T, \zeta}-\frac{1}{v} \varphi_{T p}\left(\frac{\mathrm{d} \zeta}{\mathrm{d} p}\right)_{T} . \\
\varphi_{T p} & \equiv\left(\frac{\partial v}{\partial \zeta}\right)_{T, p}
\end{aligned}
$$


is the partial specific volume with respect to the internal degree of freedom, $v$ the specific volume of the system. For the change of the internal variable as a function of pressure at constant temperature and in the internal equilibrium, one obtains, corresponding to (8),

$$
\begin{aligned}
& \left(\frac{\mathrm{d} \zeta}{\mathrm{d} p}\right)_{T}^{\mathrm{e}}=-\frac{\varphi_{T p}^{\mathrm{e}}}{\gamma_{T p}^{\mathrm{e}}} \\
& \alpha_{\zeta} \equiv \frac{1}{v}\left(\frac{\partial v}{\partial T}\right)_{p, \zeta} \text { and } \kappa_{T, \zeta} \equiv-\frac{1}{v}\left(\frac{\partial v}{\partial p}\right)_{T, \zeta}
\end{aligned}
$$

are the response functions which are measured in the arrested equilibrium. According to (8) and (16), the contribution of the internal degree of freedom to the coefficient of thermal expansion $\alpha_{\mathrm{e}}$ under conditions of internal equilibrium is

$$
\Delta^{\mathrm{e}} \alpha=\frac{1}{v} \frac{\varphi_{T p}^{\mathrm{e}} \sigma_{T p}^{\mathrm{e}}}{\gamma_{T p}^{\mathrm{e}}} .
$$

According to (17) and (19), the contribution of the internal degree of freedom to the compressibility $\kappa_{T}^{\mathrm{e}}$ of the internal equilibrium is

$$
\Delta^{\mathrm{e}} \kappa_{T}=\frac{1}{v} \frac{\left(\varphi_{T p}^{\mathrm{e}}\right)^{2}}{\gamma_{T p}^{\mathrm{e}}} \geq 0 .
$$

As can easily be seen, the differences (13b), (20), and (21) fulfil the Davies relation

$$
\Delta^{\mathrm{e}} c_{p} \Delta^{\mathrm{e}} \kappa_{T}=T v\left(\Delta^{\mathrm{e}} \alpha\right)^{2}
$$

$[7,8]$ (compare also [4]). It should be stressed that the Davies relation holds for every arbitrary internal equilibrium state $Z\left[T, p, \zeta_{\mathrm{e}}(T, p)\right]$. Hence, it does not have anything to do with the formally homologous Ehrenfest relation, which is fulfilled on the line $T_{c}\left(p_{c}\right)$ of a second-order transition. In (22), the differences $\Delta^{\mathrm{e}}$ refer to the response functions, which are measured during a quasi-static perturbation $\left[\zeta=\zeta_{\mathrm{e}}(T, p)\right.$; internal equilibrium] and during a sufficiently fast perturbation ( $\zeta_{\mathrm{e}}=$ const.; arrested equilibrium). If several internal degrees of freedom are simultaneously macroscopically relevant, (22a) is replaced by the Davies inequality [9]

$$
\Delta^{\mathrm{e}} c_{p} \Delta^{\mathrm{e}} \kappa_{T} \geq T v\left(\Delta^{\mathrm{e}} \alpha\right)^{2} .
$$

The contributions of the internal degree of freedom to the coefficient of thermal expansion or to the compressibil- ity under conditions of non-equilibrium are given by

$$
\begin{aligned}
& \Delta \alpha=\frac{1}{v} \varphi_{T_{p}} \dot{\zeta}_{p} / \dot{T}_{p} \text { or } \\
& \Delta \kappa_{T}=-\frac{1}{v} \varphi_{T p} \dot{\zeta}_{T} / \dot{p}_{T} .
\end{aligned}
$$

One must distinguish here if the process is run at constant pressure or at constant temperature. The differences (15b) and (23) do not fulfil the Davies relation (22). Since the coefficient of thermal expansion, defined by (16), must be measured at constant pressure, it would be more correct to write $\alpha_{p}$ instead of $\alpha$ and in (23) $\Delta \alpha_{p}$ instead of $\Delta \alpha$. According to the equations of equilibrium thermodynamics, we can then also define an isothermal coefficient of expansion

$$
\begin{aligned}
\alpha_{T} & \equiv-\frac{1}{v}\left(\frac{\mathrm{d} s}{\mathrm{~d} p}\right)_{T} \\
& =-\frac{1}{v}\left(\frac{\partial s}{\partial p}\right)_{T, \zeta}-\frac{1}{v} \sigma_{T p}\left(\frac{\partial \zeta}{\mathrm{d} p}\right)_{T} .
\end{aligned}
$$

In non-equilibrium, the contribution of the internal degree of freedom to this coefficient is

$$
\Delta \alpha_{T}=-\frac{1}{v} \sigma_{T p} \frac{\dot{\zeta}_{T}}{\dot{p}_{T}} .
$$

Obviously, we now have in place of (22a)

$$
\Delta c_{p} \Delta \kappa_{T}=T \nu \Delta \alpha_{p} \Delta \alpha_{T} .
$$

However, in non-equilibrium, in general, $\Delta \alpha_{p} \neq \Delta \alpha_{T}$.

It should be noted that the product $L \gamma_{T p}^{\mathrm{e}}$ has the dimension of reciprocal time if the internal variable is dimensionless (e.g., a concentration).

$$
\tau_{T p}^{\mathrm{e}}(T, p) \equiv 1 / L \gamma_{T p}^{\mathrm{e}}
$$

proves to be the so-called Debye relaxation time of the system. Analogously, one can introduce the relaxation time

$$
\tau_{T p}(T, p, \zeta)=1 / L \gamma_{T p}
$$

with

$$
\gamma_{T p} \equiv\left(\frac{\partial^{2} g}{\partial \zeta^{2}}\right)_{T, p} .
$$

In contrast to the Debye relaxation time, the relaxation time (25) also depends on the internal variable. In order to determine the internal variable as a function of time, one obtains, if the pressure $p$ and the coupling factor $L$ 
are constant, from (3) and (7) with (4) and (25) the nonlinear differential equation:

$$
\ddot{\zeta}+\frac{1}{\tau_{T p}} \dot{\zeta}=-L\left(\frac{\partial^{2} g}{\partial T \partial \zeta}\right) \dot{T} .
$$

If $\eta_{T p}$ and $\sigma_{T p}$ are not dependent on the temperature [e.g., as in the case of the Eqs. (38) and (39) in Sect. 3], we have, as in the equilibrium,

$$
-\frac{\partial^{2} g}{\partial T \partial \zeta}=\sigma_{T p} .
$$

Equation $\dot{\zeta}=0$ holds for the frozen (arrested) states. According to (3), however, $\dot{\zeta}$ does not disappear because of $a=0$ (internal equilibrium), but because of $L \rightarrow 0$. Hence, when describing a freezing process, e.g. the glass transition, $L$ must necessarily be regarded as a variable, i.e., as a function $L(T, p, \zeta)$ of the mutually independent variables of the system. In this case, the differential equation (27) is formally maintained if the relaxation time $\tau_{T p}$ is replaced by the effective relaxation time

$$
\frac{1}{\tau_{e f f}}=\frac{1}{\tau_{T p}}-\left(\frac{\partial \ln L}{\partial T}\right)_{p, \zeta} \dot{T}-\left(\frac{\partial \ln L}{\partial \zeta}\right)_{T, p} \dot{\zeta} .
$$

Thus, the effective relaxation time $\tau_{\text {eff }}$ of a freezing process under constant pressure depends not only on the independent variables $T, \zeta$, but also on their rates $\dot{T}, \dot{\zeta}$.

\section{A Gibbs Fundamental Equation for the Description of the Glass Transition}

A fundamental problem when describing the glass transition within the framework of phenomenological thermodynamics is the formulation of a suitable Gibbs fundamental equation of the melt. Here, we proceed from the simple model of the Eyring liquid [10-12]. Eyring regards liquids as a mixture of molecules and vacancies. What freezes during the glass transition of a melt, is without doubt the diffusive translational motion of the molecules. The concentration of the vacancies or the volume fraction of the vacancies (the relative free volume) of the Eyring liquid can serve as a measure of the intensity of the diffusive translational motion. In this case, the diffusion process should not be understood as a hopping of the molecules into holes of the same size. In a relatively compact melt, the diffusion process is mainly a cooperative process, for which a considerably smaller vacancy size is sufficient [13]. We thus proceed from a mixture whose species differ widely in their volumes.
According to Flory $[14,15]$, one can write for the free enthalpy $G$ of a mixture of molecules with two different volumes:

$$
\begin{aligned}
& G= G_{0}+R T\left[N_{1} \ln \frac{N_{1}}{N_{1}+\rho N_{2}}\right. \\
&\left.+N_{2} \ln \frac{\rho N_{2}}{N_{1}+\rho N_{2}}\right]+G_{\mathrm{E}} . \\
& \rho \equiv v_{2} / v_{1}>1
\end{aligned}
$$

is the ratio of the molar volumes of the two mixing partners, $N_{1}$ and $N_{2}$ their mole numbers, and $R$ the gas constant. With respect to the Eyring liquid, $N_{1}$ designates the mole number of the vacancies* and $N_{2}$ the mole number of the material particles (molecules of a low-molecularweight substance or mobile units of a flexible polymer). The standard term $G_{0}$ is solely determined by the material particles, as the vacancies in the pure state do not possess a chemical potential $\mu$ :

$$
G_{0}=\mu_{2}^{0} N_{2} ; \quad \mu_{1}^{0}=0 .
$$

For the deduction of an expression for the excess free enthalpy $G_{\mathrm{E}}$, we proceed from a simple mixture as defined by Guggenheim [15]:

$$
G_{\mathrm{E}}=z \Delta w \frac{N_{1} N_{2}}{N_{1}+N_{2}} .
$$

Here, however, we substitute with

$$
z N_{i} \rightarrow o_{i} N_{i}
$$

the coordination number $z$ by the contact surfaces $o_{i}$ per mole of the mixing partners [16]. It is obvious that the interaction between partners of different sizes and shapes can be described much better by the surfaces $o_{i}$ than by the mere number $z$ of the nearest neighbours. Hence, the excess free enthalpy should amount to

$$
G_{\mathrm{E}}=\Delta w \frac{o_{1} N_{1} o_{2} N_{2}}{o_{1} N_{1}+o_{2} N_{2}} .
$$

In

$$
\Delta w \equiv w_{12}-\frac{1}{2}\left(w_{11}+w_{22}\right)
$$

* The mole number and the number of vacancies are fictitious quantities, since the vacancies have no mass and, continuously smeared, do not have an individuality. According to (34) and (37), on the other hand, a real physical significance is attributed to the relative free volume $\varphi$, introduced instead of $N_{1}$ further down, and to the internal variable $\zeta$. 
$w_{22}$ describes the binding energy between two adjacent material particles and $w_{11}=0$ the binding energy between two vacancies. $w_{12}$ can be interpreted as the binding energy between two material particles separated by vacancies.

We approximatively assume that the excess free enthalpy $G_{\mathrm{E}}$ neither depends on the temperature nor the pressure. The temperature independence means that we confine ourselves to a regular mixture with

$$
G_{\mathrm{E}}=H_{\mathrm{E}}, \quad S_{\mathrm{E}}=0 .
$$

The pressure independence allows an equation of the molar volumes $v_{i}$ with the partial molar volumes $\bar{v}_{i}$. In place of the mole numbers $N_{i}$, we can then easily introduce the volume fractions

$$
\varphi_{1} \equiv \frac{\bar{v}_{1} N_{1}}{\bar{v}_{1} N_{1}+\bar{v}_{2} N_{2}}=\frac{N_{1}}{N_{1}+\rho N_{2}} ; \varphi_{2}=1-\varphi_{1}
$$

as variables of composition. In the following, we will use the volume fraction $\varphi \equiv \varphi_{1}$ of the vacancies (the relative free volume) as the independent variable of the composition. Furthermore, we will refer all the extensive quantities to the mole number $N_{2}$ of the material particles and once again denote these molar quantities with small letters $G / N_{2}=g, V / N_{2}=v, C_{p} / N_{2}=c_{p}$ etc. $(29-32)$ then yield as the Gibbs fundamental equation of the melt interspersed with vacancies:

with

$$
g=g_{0}+R T\left[\frac{\rho \varphi}{1-\varphi} \ln \varphi+\ln (1-\varphi)\right]+g_{\mathrm{E}}
$$

$$
g_{0}=\mu_{2}^{0}(T, p)
$$

and

$$
\begin{aligned}
& g_{\mathrm{E}}=h_{\mathrm{E}}=h_{c} \frac{\rho \varphi}{1-\varphi+\lambda \varphi} . \\
& h_{c} \equiv o_{1} \Delta w
\end{aligned}
$$

is the energy required to generate one mole of vacancies in the vacancy-free melt.

$$
\lambda \equiv \frac{o_{1}}{o_{2}} \rho, \text { i.e., } \frac{o_{1}}{o_{2}}=\lambda \frac{v_{1}}{v_{2}},
$$

is a geometric factor which not only considers the different sizes but also the different shapes of the mixing partners. As dimensionless internal variable, we will finally introduce the ratio $\zeta=N_{1} / N_{2}$ of the mole number of the vacancies to the mole number of the material particles. The internal variable, which is often rather vaguely re- ferred to as the "ordering parameter" in the literature (e.g., compare [3], [8], [17]), thus acquires a concrete physical meaning. With the relative free volume $\varphi$, we find the interrelation

$$
\zeta=\frac{\rho \varphi}{1-\varphi} ; \quad \mathrm{d} \zeta=\frac{\rho}{(1-\varphi)^{2}} \mathrm{~d} \varphi .
$$

According to (2) and (33), the entropy and enthalpy of the system are given by

$$
\begin{aligned}
& s=-\left(\frac{\partial g_{0}}{\partial T}\right)_{p}-R\left[\frac{\rho \varphi}{1-\varphi} \ln \varphi+\ln (1-\varphi)\right], \\
& h=h_{0}+h_{\mathrm{E}} \quad \text { with } \quad h_{0}=g_{0}-T\left(\frac{\partial g_{0}}{\partial T}\right)_{p} .
\end{aligned}
$$

One obtains for the volume

$$
v=v_{1} \zeta+v_{2}=\frac{v_{2}}{1-\varphi} .
$$

The vacancies are not of importance in the response functions $c_{p, \zeta}, \alpha_{\zeta}, \kappa_{T, \zeta}$ of the arrested equilibrium. According to (12) and (35), one obtains, for example, for the heat capacity of the arrested equilibrium

$$
c_{p, \zeta}=-T\left(\frac{\partial^{2} g_{0}}{\partial T^{2}}\right)_{p} .
$$

Equations (33) and (34) yield for the quantities (5), (18), and (26), which determine the dynamics of the system

$$
\begin{aligned}
& \eta_{T p}=h_{c}\left(\frac{1-\varphi}{1-\varphi+\lambda \varphi}\right)^{2}, \\
& \sigma_{T p}=-R\left[\ln \varphi+\left(1-\frac{1}{\rho}\right)(1-\varphi)\right], \\
& \varphi_{T p}=v_{1}=\frac{1-\varphi}{\rho} v
\end{aligned}
$$

and

$$
\gamma_{T p}=\left(\frac{\partial \eta_{T p}}{\partial \zeta}\right)_{T, p}-T\left(\frac{\partial \sigma_{T p}}{\partial \zeta}\right)_{T, p}
$$

with

$$
\left(\frac{\partial \eta_{T p}}{\partial \zeta}\right)_{T, p}=-\frac{2 h_{c} \lambda}{\rho}\left(\frac{\eta_{T p}}{h_{c}}\right)^{3 / 2},
$$




$$
\left(\frac{\partial \sigma_{T p}}{\partial \zeta}\right)_{T, p}=-\frac{R}{\rho} \frac{(1-\varphi)^{2}}{\varphi}\left(1-\varphi+\frac{\varphi}{\rho}\right) .
$$

In consideration of the equilibrium condition (6a), one can also write for the equilibrium value of $\gamma_{T p}$ :

$$
\begin{aligned}
\gamma_{T p}^{\mathrm{e}}= & R T \frac{1-\varphi_{\mathrm{e}}}{\rho} \\
& \cdot\left[\frac{2 \lambda}{1-\varphi_{\mathrm{e}}+\lambda \varphi_{\mathrm{e}}}\left[\ln \varphi_{\mathrm{e}}+\left(1-\frac{1}{\rho}\right)\left(1-\varphi_{\mathrm{e}}\right)\right]\right. \\
& \left.+\frac{1-\varphi_{\mathrm{e}}}{\varphi_{\mathrm{e}}}\left(1-\varphi_{\mathrm{e}}+\frac{\varphi_{\mathrm{e}}}{\rho}\right)\right]
\end{aligned}
$$

As already mentioned, $\tau_{T p}^{\mathrm{e}}=1 / L \gamma_{T p}^{\mathrm{e}}$ is the Debye relaxation time of the system.

In the internal equilibrium, the liquid interspersed with vacancies (33) is stable up to $T \rightarrow 0$. However, the liquid is not capable of holding an arbitrary number of vacancies. With increasing temperature, a critical point $\left(T_{\max } ; \varphi_{\max }^{\mathrm{e}}\right)$ is reached, after which the system becomes unstable with $\gamma_{T p}^{\mathrm{e}}<0$.

\section{The Glass Transition in the "Simple Freezing-in Model"}

In the "simple freezing-in model" (Sect. 1), the glass transition region is reduced to the so-called glass temperature $T_{\mathrm{g}}$. The glass transition appears as a discontinuous process in which an equilibrium value $\zeta_{\mathrm{e}}(T)$ of the internal variable of the liquid state is abruptly subjected to the arrest $\zeta_{\mathrm{e}}=$ const. As a result, the response functions undergo a precipitous change in the amounts given by the equilibrium differences (13b), (20), and (21).

With (39), (40), and (42), (20) now leads to

$$
\Delta^{\mathrm{e}} \alpha=-\frac{1}{T} \frac{\varphi_{\mathrm{e}}}{\frac{2 \lambda \varphi_{\mathrm{e}}}{1-\varphi_{\mathrm{e}}+\lambda \varphi_{\mathrm{e}}}+\frac{\left(1-\varphi_{\mathrm{e}}\right)\left(1-\varphi_{\mathrm{e}}+\frac{\varphi_{\mathrm{e}}}{\rho}\right)}{\ln \varphi_{\mathrm{e}}+\left(1-\frac{1}{\rho}\right)\left(1-\varphi_{\mathrm{e}}\right)}} .
$$

According to (13b) and (20), we have further

$$
\Delta^{\mathrm{e}} c_{p}=v T \Delta^{\mathrm{e}} \alpha \frac{\sigma_{T p}^{\mathrm{e}}}{\varphi_{T p}^{\mathrm{e}}},
$$

i.e., with (39), (40), and (43)

$$
\Delta^{\mathrm{e}} c_{p}=\frac{R \rho \varphi_{\mathrm{e}}}{1-\varphi_{\mathrm{e}}}
$$

$$
\frac{\ln \varphi_{\mathrm{e}}+\left(1-\frac{1}{\rho}\right)\left(1-\varphi_{\mathrm{e}}\right)}{\frac{2 \lambda \varphi_{\mathrm{e}}}{1-\varphi_{\mathrm{e}}+\lambda \varphi_{\mathrm{e}}}+\frac{\left(1-\varphi_{\mathrm{e}}\right)\left(1-\varphi_{\mathrm{e}}+\frac{\varphi_{\mathrm{e}}}{\rho}\right)}{\ln \varphi_{\mathrm{e}}+\left(1-\frac{1}{\rho}\right)\left(1-\varphi_{\mathrm{e}}\right)}} .
$$

These equations hold for every arbitrary internal equilibrium state of the system (33), i.e., with the assumption $\zeta_{\mathrm{g}}=\zeta_{\mathrm{e}}\left(T_{\mathrm{g}}\right)$ also at the glass temperature $T_{\mathrm{g}}$. If $\Delta^{\mathrm{e}} \alpha\left(T_{\mathrm{g}}\right)$, $\Delta^{\mathrm{e}} c_{p}\left(T_{\mathrm{g}}\right), \rho$, and $T_{\mathrm{g}}$ are known, the relative free volume $\varphi_{\mathrm{e}}\left(T_{\mathrm{g}}\right)$ and the interaction parameter $\lambda$ can be determined from (43) and (44).

Using equivalent equations (under the precondition $\varphi_{\mathrm{e}} \ll 1$ and omission of $1 / \rho$ as compared to 1 in Eq. (39)), Kanig [16] determined the volume fraction $\varphi_{\mathrm{g}}^{\mathrm{e}} \equiv \varphi_{\mathrm{e}}\left(T_{\mathrm{g}}\right)$ and the interaction parameter $\lambda$ for eleven different organic polymers with experimental data for $\Delta \alpha, \Delta c_{p}$, and $T_{\mathrm{g}}$. For these polymers, he found on average

$$
\left\langle\varphi_{\mathrm{g}}^{\mathrm{e}}\right\rangle=0.0235 \pm 0.0050 \text {, }
$$

$$
\langle\lambda\rangle=3.15 \pm 0.35 \text {. }
$$

The mean value (45) agrees very well with the value $\varphi_{\mathrm{g}}^{\mathrm{e}} \approx 0.025$ determined by Williams, Landel, Ferry [18] from viscosimetric data. Equation (45) seems to confirm the thesis established by Fox and Flory [19], according to which liquids are in a state of equal free volume at the glass temperature. Equation (45) also seems to confirm the rule of Simha and Boyer [20], according to which $T_{\mathrm{g}} \Delta \alpha=$ const. should always hold at the glass temperature. However, Kanig [16] already noticed systematic deviations from the mean value (45). Polymers composed of more flexible chain molecules, for example, lead to a smaller $\varphi_{\mathrm{g}}^{\mathrm{e}}$-value than polymers composed of stiffer chain molecules. Wrasidlo [21] finally showed on the basis of the data for 63 polymers that an "iso-free volume state" is out of the question at $T_{\mathrm{g}}$. From the viewpoint of the thermodynamics of irreversible processes, one should add that an equilibrium value $\varphi_{\mathrm{e}}$ can never freeze in at a finite cooling rate. Moreover, the non-equilibrium value $\varphi>\varphi_{\mathrm{e}}$, which practically freezes in at a temperature $T_{\mathrm{g}}^{\prime}<T_{\mathrm{g}}$, depends on the cooling rate $\beta$ (Figure 2).

Together with an adequate expression for $\Delta^{\mathrm{e}} \kappa_{T}$, the differences (43) and (44) fulfil the Davies relation (22a). 
Hence, in the "simple freezing-in model" the Davies relation in the form (22a) or also in the form (22b) appears as a characteristic of the glass transition and, as such, is also designated as the Prigogine-Defay relation (e.g., compare [2], [22-24]. One should notice here that the Davies relation can, strictly speaking, never become valid at a finite cooling rate, since we are dealing with an irreversible process subject to a non-vanishing entropy production in the whole interval $\left\langle T_{\mathrm{g}}^{\prime \prime}, T_{0}\right\rangle$ (Figure 9). The Davies relation approximately holds for the states $Z$ $(T, p, \zeta)$ in the interval $\left\langle T_{\mathrm{F}}, T_{0}\right\rangle$, as in this region the response functions still approximately follow the equilibrium curves with $\zeta \approx \zeta_{\mathrm{e}}(T, p)$. Below $T_{\mathrm{F}}$, however, the internal degree of freedom provides the "dynamic" contributions (15b) and (23) to the response functions, which do not comply with the Davies relation. These contributions continuously diminish from $T_{\mathrm{F}}$ until they approximately disappear at $T_{\mathrm{g}}^{\prime}$ and finally completely disappear at $T_{\mathrm{g}}^{\prime \prime}$ (Figs. 7 and 8). With regard to the glass transition, the Davies or Prigogine-Defay relation can only attain significance upon quasi-static cooling with $\dot{T}=\beta \rightarrow 0$. According to the statements in the next section, this is the case at the temperature $T_{\mathrm{g}}^{\prime \prime} \equiv T_{\infty}$, which is usually some ten degrees below the glass temperature.

\section{The Glass Transition as an Irreversible Process}

In the thermodynamics of irreversible processes, the system temperature $T$ is defined by

$$
T(s, v, \zeta)=\left(\frac{\partial u}{\partial s}\right)_{v, \zeta}
$$

( $u$ : specific internal energy of the system). It reflects all the internal degrees of freedom of the system which are in internal equilibrium. The macroscopically relevant internal degree of freedom $\zeta$, on the other hand, must be regarded as being arrested with $\zeta=$ const. If $\zeta$ is assigned to the diffusive translational motion of the molecules of a liquid in non-equilibrium, one must, therefore, expect a considerable difference between the system temperature and that temperature $T^{*}$ at which the heat exchange of the system with its surroundings takes place. According to Meixner [25], the differentiation between a thermostatic temperature $T$ and a thermodynamic temperature $T^{*}$ is even generally imperative in the thermodynamics of irreversible processes. This completely corresponds to the differentiation between the hydrostatic and the hydrodynamic pressures in hydromechanics. In the following, the differentiation between $T$ and $T^{*}$ finally proves to be essential for a correct formulation of the boundary conditions. $T=T^{*}$ naturally holds in the internal as well as in the arrested equilibrium.

In non-equilibrium, the change in entropy $s$ with time is separated into two additive parts:

$$
\dot{s}=\frac{\mathrm{d}_{a} s}{\mathrm{~d} t}+\frac{\mathrm{d}_{i} s}{\mathrm{~d} t} .
$$

$\mathrm{d}_{a} s / \mathrm{d} t$ describes the amount of entropy exchanged by the system with its surroundings per time and mass unit. $\mathrm{d}_{i} s / \mathrm{d} t \geq 0$ is the entropy produced in the interior of the system per time and mass unit [26]. If one distinguishes between the static temperature $T$ and the dynamic temperature $T^{*}$, one obtains

$$
\frac{\mathrm{d}_{a} s}{\mathrm{~d} t}=\frac{1}{T^{*}} \dot{q} .
$$

$\dot{q}$ is the heat exchanged by the system with its surroundings at the temperature $T^{*}$ per time and mass unit. If we neglect the possible difference between the hydrostatic and the hydrodynamic pressures and assume $p=$ const., we have

$$
\dot{q}=\dot{h},
$$

whereby (36) with (5) and (12) leads to

$$
\dot{h}=c_{p, \zeta} \dot{T}+\eta_{T p} \dot{\zeta} .
$$

According to (10) with (5) and (12), the change in entropy with time is given by

$$
\dot{s}=\frac{1}{T} c_{p, \zeta} \dot{T}+\sigma_{T p} \dot{\zeta} .
$$

Hence, according to (47), (48), and (49), the entropy produced in the interior of the system must be

$$
\frac{\mathrm{d}_{i} s}{\mathrm{~d} t}=c_{p, \zeta}\left(\frac{1}{T}-\frac{1}{T^{*}}\right) \dot{T}+\left(\sigma_{T p}-\frac{1}{T^{*}} \eta_{T p}\right) \dot{\zeta} .
$$

In the thermodynamics of irreversible processes, the product $T\left(\mathrm{~d}_{i} s / \mathrm{d} t\right)$ now always appears in a bilinear form of the effective "fluxes" and "forces" [26]. Therefore, we can conclude from (50) that, in the case of a differentiation between the static and the dynamic temperatures, Eq. (3) will be replaced by the dynamic laws of the form

$$
\begin{aligned}
\dot{T}= & L_{11} c_{p, \zeta} \frac{T^{*}-T}{T^{*}} \\
& +L_{12} T\left(\sigma_{T p}-\frac{1}{T^{*}} \eta_{T p}\right),
\end{aligned}
$$




$$
\begin{aligned}
\dot{\zeta}= & L_{21} c_{p, \zeta} \frac{T^{*}-T}{T^{*}} \\
& +L_{22} T\left(\sigma_{T_{p}}-\frac{1}{T^{*}} \eta_{T p}\right) .
\end{aligned}
$$

The matrix of the coefficients $L_{i k}$ is positive definite because of $\mathrm{d}_{i} s / \mathrm{d} t \geq 0$. If we ignore with $L_{12}=L_{21}=0$ the possible interference effects, we obtain the simple dynamic laws

$$
\begin{aligned}
& \dot{T}=L_{11} c_{p, \zeta} \frac{T^{*}-T}{T^{*}}, \\
& \dot{\zeta}=L_{22} \frac{T}{T^{*}}\left(T^{*} \sigma_{T p}-\eta_{T_{p}}\right) .
\end{aligned}
$$

With a constant cooling or heating rate, we also have the differential equation

$$
\dot{T}^{*}=\beta=\text { const. }
$$

For the internal variable $\zeta$, there is, as before, a simple dynamic law of the form (3):

$$
\dot{\zeta}=L_{22} a^{*} \text {. }
$$

However, the affinity $a$ must now be replaced by the "dynamic" affinity

$$
a^{*} \equiv \frac{T}{T^{*}}\left(T^{*} \sigma_{T p}-\eta_{T p}\right) .
$$

Between $a$ and $a^{*}$, we find the interrelation

$$
a^{*}=a+\eta_{T p} \frac{T^{*}-T}{T^{*}} .
$$

According to Tool [27] (see also [8]), one can further introduce a fictive temperature

$$
T_{\mathrm{f}} \equiv \frac{\eta_{T p}}{\sigma_{T p}} .
$$

This is the temperature which the system in a non-equilibrium state $Z(T, \zeta)$ would have if it were in internal equilibrium [see Eq. (6)]. With the fictive temperature (58), one can also write instead of (4) and (56)

$$
a=\sigma_{T p}\left(T-T_{\mathrm{f}}\right)
$$

and

$$
a^{*}=T \sigma_{T p}\left(T^{*}-T_{\mathrm{f}}\right) / T^{*} .
$$

The functional dependence of the coefficients $L_{i k}=L_{i k}(T, p, \zeta)$ cannot be derived in the macroscopicphenomenological theory. In this respect, one rather depends on ad-hoc formulations. $L_{22}$ has the dimension fluidity/volume. With respect to the description of the glass transition, it is reasonable to assume a proportionality

$$
L_{22} \sim 1 / \eta
$$

( $\eta$ : viscosity of the melt). In order to describe the temperature dependence of the viscosity, we proceed from the so-called Vogel-Fulcher-Tammann-Hesse equation $[28,29]$

$$
\ln \eta=\ln \eta^{\prime}+\frac{c}{T-T_{\infty}}
$$

and correspondingly set

$$
\begin{aligned}
& L_{22}(t)= \\
& L_{22}(0) \exp \left[c\left(\frac{1}{T_{0}^{*}-T_{\infty}}-\frac{1}{T^{*}-T_{\infty}}\right)\right] .
\end{aligned}
$$

Here and in the following, the subscript " 0 " refers to the initial state. $T_{\infty}$ is the Vogel temperature. At the same time, $T_{\infty}$ and $c$ are the invariants of the so-called Williams-Landel-Ferry equation $[18,28,29]$. With (61), (53) leads to $\dot{\zeta} \rightarrow 0$ if $\eta \rightarrow \infty$. Hence, the internal degree of freedom freezes completely at the Vogel temperature. $T_{\infty}$ is identical with the temperature denoted with $T_{\mathrm{g}}^{\prime \prime}$ in Figure 1 .

The boundary conditions should particularly be considered when determining the coefficient $L_{11}$. Proceeding from an internal equilibrium state $Z\left(T_{0}^{*}, \zeta_{\mathrm{e}}\left(T_{0}^{*}\right)\right)$, we cool the melt under constant pressure at the constant rate $\dot{T}^{*}=\beta$. In the initial state, we have $T_{0}=T_{\mathrm{f}}^{0}=T_{0}^{*}$ and $\dot{T}_{0}=\dot{T}_{\mathrm{f}}^{0}=0, \dot{T}_{0}^{*}=\beta$. Approaching the Vogel temperature, the internal degree of freedom is arrested with a nonequilibrium value $a^{*}=a \neq 0$. Hence, $T^{*}=T \neq T_{\mathrm{f}}$ and $\dot{T}^{*}=\dot{T}=\beta, \dot{T}_{\mathrm{f}}=0$ must hold in the limit $T^{*} \rightarrow T_{\infty}$. Moreover, according to (8) and (14),

$$
\lim _{T^{*} \rightarrow T_{0}^{*}} \frac{\dot{\zeta}}{\dot{T}}=\left(\frac{\mathrm{d} \zeta}{\mathrm{d} T}\right)_{p}^{0}
$$

must hold if the transition from the internal equilibrium to the non-equilibrium is supposed to proceed continuously in $T$ and $\zeta$ at the beginning of the process. With (8), (52), (55), and (60), this leads to

$$
\lim _{T^{*} \rightarrow T_{0}^{*}} \frac{L_{22} T \sigma_{T_{p}}\left(T^{*}-T_{\mathrm{f}}\right)}{L_{11} c_{p, \zeta}\left(T^{*}-T\right)}=\frac{\sigma_{T p}^{0}}{\gamma_{T p}^{0}}
$$

and because of

$$
\lim _{T^{*} \rightarrow T_{0}^{*}} \frac{T^{*}-T_{\mathrm{f}}}{T^{*}-T}=1
$$


to

$$
L_{11}(0)=\frac{T_{0}}{c_{p, \zeta}^{0}} L_{22}(0) \gamma_{T p}^{0}=\frac{T_{0}}{c_{p, \zeta}^{0} \tau_{T p}^{0}} .
$$

The boundary conditions for $T^{*} \rightarrow T_{\infty}$ are satisfied if one first generalizes (62) as

$$
L_{11}=\frac{T}{c_{p, \zeta} \tau_{T p}}
$$

and replaces the relaxation time $\tau_{T p}$ by the effective relaxation time (28). According to (28) and (61), one obtains for $\beta<0$ :

$$
\begin{aligned}
\frac{1}{\tau_{e f f}} & =\frac{1}{\tau_{T p}}-\frac{c \beta}{\left(T^{*}-T_{\infty}\right)^{2}} \\
& =\frac{1}{\tau_{T p}}+\frac{c|\beta|}{\left(T^{*}-T_{\infty}\right)^{2}} .
\end{aligned}
$$

In order to maintain the limiting value (62), we should then set

$$
\begin{aligned}
L_{11}= & \frac{T}{c_{p, \zeta}}\left[L_{22} \gamma_{T_{p}}+c|\beta|\right. \\
& \left.\cdot\left(\frac{1}{\left.T^{*}-T_{\infty}\right)^{2}}-\frac{1}{\left(T_{0}^{*}-T_{\infty}\right)^{2}}\right)\right] .
\end{aligned}
$$

Equation (63) can also be used for $\beta>0$, since $L_{11}>0$ is always valid regardless of the sign of $\beta$.

The differential equations $(52-54)$ with the phenomenological coefficients (61) and (63), and the thermodynamic coefficients (38-41) are thus the determining equations for the glass transition. Numerical solutions of these equations are represented in the following figures. For the constants of the equations, we will choose values which approximately apply for polystyrene:

$$
h_{c}=10 \mathrm{~kJ} / \mathrm{mol}, \rho=10, \lambda=3,
$$

according to [16],

$$
T_{\infty}=340 \mathrm{~K}, c=400 \mathrm{~K},
$$

according to [28], and with $T_{0}=T_{0}^{*}=400 \mathrm{~K}$ :

$$
L_{22}(0)=0.0001 \mathrm{~mol} / \mathrm{J} \mathrm{sec} \text {. }
$$

The heat capacity $c_{p, \zeta}$ of the arrested equilibrium only plays a role in Figs. 6 and 9. There, we assume

$$
\begin{aligned}
& c_{p, \zeta}=0.75\left(T-T_{0}\right)+c_{p, \zeta}\left(T_{0}\right) \quad \text { with } \\
& c_{p, \zeta}\left(T_{0}\right)=150 \mathrm{~J} / \mathrm{mol} \mathrm{K} .
\end{aligned}
$$

Strictly speaking, the quantities (64a) are naturally not constant. The values assumed with (64a) have the effect that the melt in internal equilibrium already loses its stability towards higher temperatures at

$$
T_{\max }^{\mathrm{e}}=456 \mathrm{~K}, \quad \varphi_{\max }^{\mathrm{e}}=0.125 .
$$

However, this should at least not qualitatively influence the glass transition to be described.

In Fig. 2, the relative free volume $\varphi$ is represented as a function of the temperature $T^{*}$ for different cooling rates $\dot{T}^{*}=\beta<0$. It becomes obvious that at normal cooling rates the internal degree of freedom freezes practically at approximately $30 \mathrm{~K}$ above the Vogel temperature $T_{\infty}=340 \mathrm{~K}$. The frozen value of $\varphi$ is higher the faster the cooling. It is much higher than the value $\varphi_{\mathrm{e}}\left(T_{\infty}\right)=0.01679$, which would be reached upon quasi-

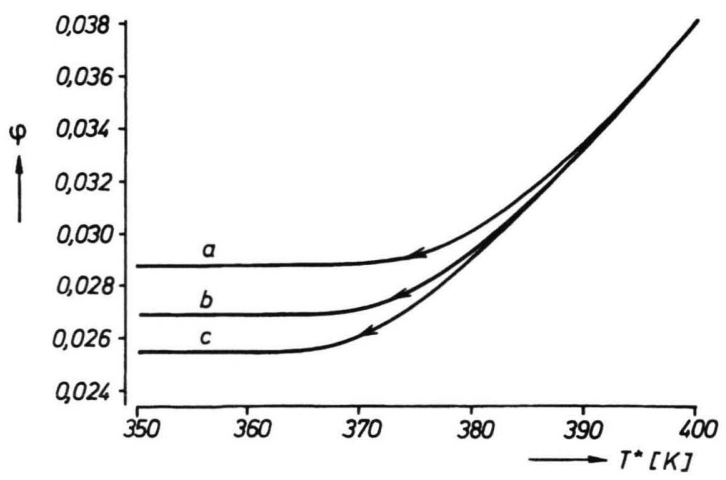

Fig. 2. Relative free volume $\varphi$ as a function of the dynamic temperature $T^{*}$ when cooling at different constant rates $T^{*}=\beta$ according to (52-54) with (61), (63), (64) and (34), (38), (39). a) $\beta=-5 \mathrm{~K} / \mathrm{min}$; b) $\beta=-1 \mathrm{~K} / \mathrm{min}$; c) $\beta=-0.2 \mathrm{~K} / \mathrm{min}$. According to (6), (38), (39), the equilibrium value of the relative free volume is at the initial temperature $T_{0}^{*}=400 \mathrm{~K}: \varphi_{\mathrm{e}}=0.038017$, at $T^{*}=370 \mathrm{~K}: \varphi_{\mathrm{e}}=0.025343$, and at the Vogel temperature $T_{\mathrm{g}}^{\prime \prime} \equiv T_{\infty}=340 \mathrm{~K}: \varphi_{\mathrm{e}}=0.016791$.

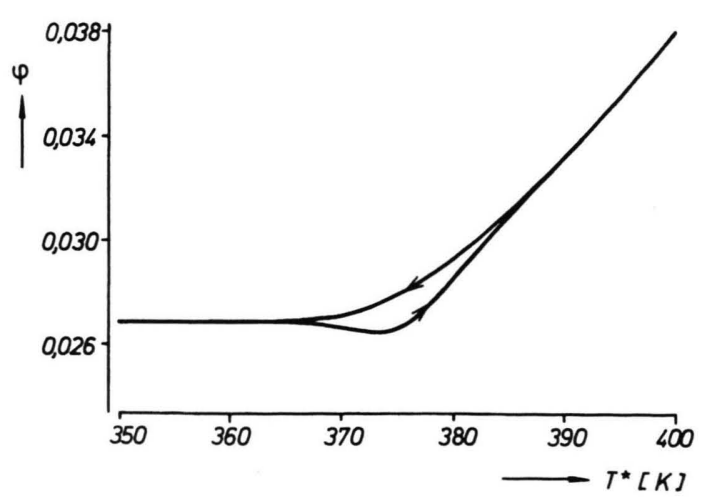

Fig. 3. Relative free volume $\varphi$ as a function of the dynamic temperature $T^{*}$ when cooling at the rate $\beta=-1 \mathrm{~K} / \mathrm{min}$ down to $T^{*}=350 \mathrm{~K}$ and subsequently heating at the rate $\beta=+1 \mathrm{~K} / \mathrm{min}$. 
static cooling $(\beta \rightarrow 0)$ at the Vogel temperature. If the melt is cooled down to the freezing range at the rate $\beta<0$ and then immediately heated up again at the same rate $|\beta|$, the free volume goes through a hysteresis cycle (Figure 3 ). The cause for this can be found in the affinity $a^{*}$, which with $\dot{\zeta}<0$ necessarily assumes negative values when cooled. If one heats up again, $a^{*}$ at first stays negative. $\zeta$ and $\varphi$ decrease further until $a^{*}$ becomes positive. Upon heating, $a^{*}$ must necessarily approach the equilibrium value $a^{*}=0$ from the positive side. Therefore, $a^{*}$ first intersects the equilibrium axis and passes through a maximum within the positive range in order to finally reach the equilibrium value $a^{*}=0$ "from above" (Figure 4).

According to (52), if $\dot{T}^{*}$ and $\dot{T}$ have the same sign, the static temperature $T$ is always above the dynamic temperature $T^{*}$ when cooling and always below it when heating. The temperature of the system retards with respect to the temperature of the surroundings. The maximum distance $\left|T^{*}-T\right|$ is smaller, the smaller the cooling or heating rate. In the limit $\beta \rightarrow 0$ we, of course, have $T^{*}-T=0$. Upon cooling, because of the proximity to the internal equilibrium, $T$ first follows the fictive temperature $T_{\mathrm{f}}$ [Eq. (59) with $a=0$ ] and then turns towards the dynamic temperature (arrested equilibrium with $T=$ $T^{*} \neq T_{\mathrm{f}}$ ) (Figure 5). The fictive temperature $T_{\mathrm{f}}$ freezes in with $\varphi$ during the glass transition. Upon heating, $T_{\mathrm{f}}$, like $\varphi$, passes through a hysteresis cycle (Figure 5 ). The behaviour of the static temperature $T$ is, of course, completely a result of our formulation (63). Except for "pathological" cases, however, a different behaviour does not seem to be possible - at least qualitatively.

In Fig. 6, the heat capacity $c_{p}$, according to (15), is represented for different cooling rates. The faster the cooling, the faster the curves separate from the equilibrium curve, and the faster the melt freezes. The width of the freezing range increases with the cooling rate. The bar on the right of the figure indicates the jump $\Delta^{\mathrm{e}} c_{p}$, which the heat capacity would suffer upon quasi-static cooling $(\beta \rightarrow 0)$ at the Vogel temperature $T_{\infty}=340 \mathrm{~K}$. Only this quantity satisfies the Davies relation (22). It is considerably smaller than the step in the heat capacity which one finds in the freezing range about $30 \mathrm{~K}$ above the Vogel temperature in the case of normal cooling rates.

If the melt is cooled down to the freezing range at the rate $\beta<0$ and then heated up again at the same rate $|\beta|$, the heat capacity $\Delta c_{p}$, according to (15b), first passes through a minimum and then through a maximum during the heating process (Figure $7 \mathrm{~b}$ ). This effect is also entirely due to the affinity. According to (15b), we have

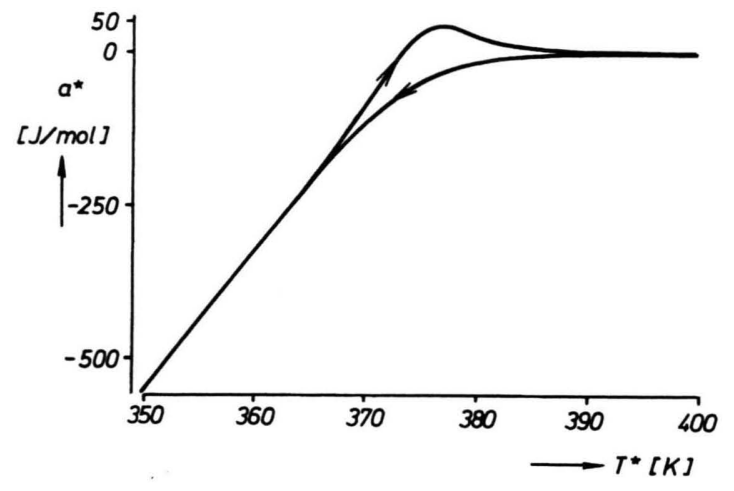

Fig. 4. Affinity $a^{*}$, according to (56), as a function of the dynamic temperature $T^{*}$ when cooling and heating at the rate $|\beta|=1 \mathrm{~K} / \mathrm{min}$.

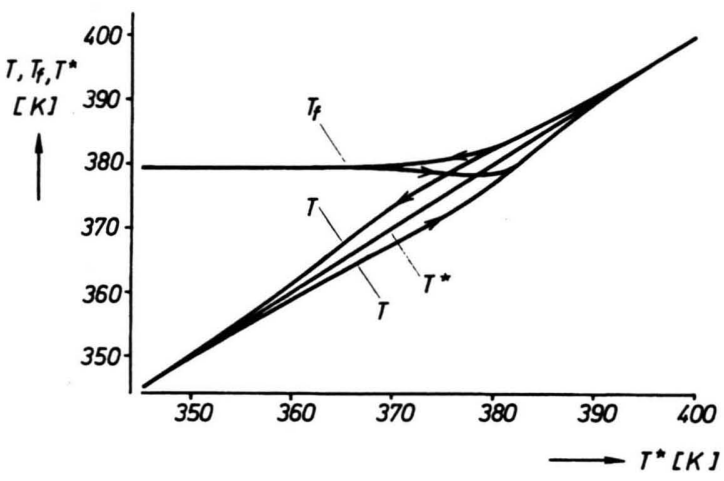

Fig. 5. Dynamic temperature $T^{*}$, static temperature $T$ and fictive temperature $T_{\mathrm{f}}$ as a function of the dynamic temperature $T^{*}$ when cooling down to $T^{*}=345 \mathrm{~K}$ and subsequently heating. $|\beta|=5 \mathrm{~K} / \mathrm{min}$.

with (55)

$$
\Delta c_{p}=L_{22} T \sigma_{T p} a^{*} / \dot{T} .
$$

As long as $a^{*}<0$ holds with $\dot{T}>0, \Delta c_{p}$ decreases upon heating. If the affinity passes through a maximum (Fig. 4), $\Delta c_{p}$ - if $T$ increases monotonously - also passes through a maximum. If one heats up at a lower rate $\dot{T}^{*}<|\beta|$, the glass thaws in a lower, narrower temperature range. The minimum becomes more pronounced while the maximum appears weakened (Figure $7 a$ ). If one heats at a higher rate $\dot{T}^{*}>|\beta|$, the thawing process takes place in a higher, broader temperature range. The minimum of the heat capacity reduces while the maximum increases (Figure 7c).

If the melt is cooled down to the freezing range at the rate $\beta<0$, it reaches a relative free volume $\varphi\left(T^{*}, \beta\right)$ at the temperature $T^{*}$, which is greater than the equilibri- 


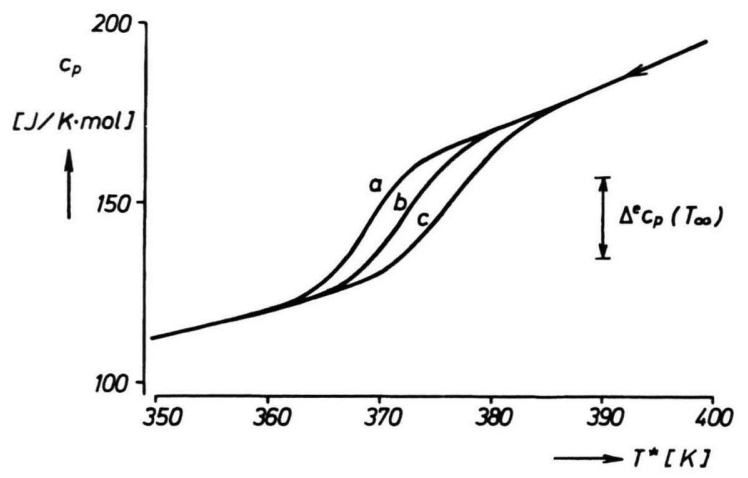

Fig. 6. Heat capacity per mole of the material particles $c_{p}$, according to (15), as a function of the dynamic temperature $T^{*}$ when cooling at the rates a) $\beta=-0.2 \mathrm{~K} / \mathrm{min}$; b) $\beta=-1 \mathrm{~K} / \mathrm{min}$; c) $\beta=-5 \mathrm{~K} / \mathrm{min} . \Delta^{\mathrm{e}} c_{p}\left(T_{\infty}\right)$ : jump in the heat capacity which, according to (13), occurs upon quasi-static cooling at the Vogel temperature $T_{\infty}=340 \mathrm{~K}$.

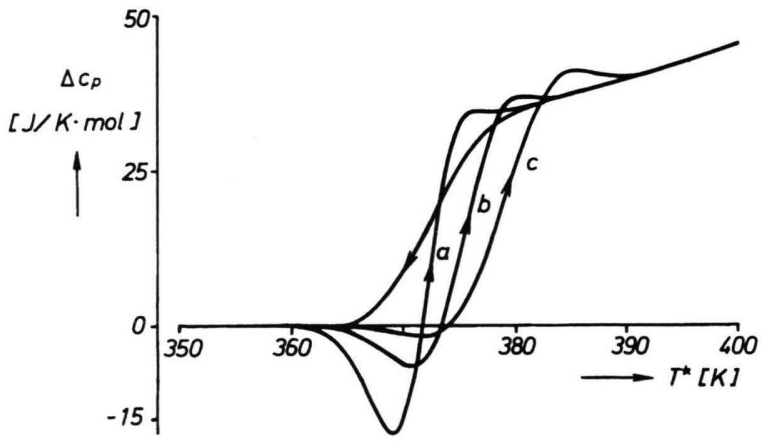

Fig. 7. Contribution $\Delta c_{p}$ of the vacancies to the heat capacity, according to (15b), when cooling from $400 \mathrm{~K}$ down to $350 \mathrm{~K}$ at the rate $\beta=-1 \mathrm{~K} / \mathrm{min}$ and subsequently heating at the rates a) $\beta=0.2 \mathrm{~K} / \mathrm{min}$; b) $\beta=1 \mathrm{~K} / \mathrm{min}$; c) $\beta=5 \mathrm{~K} / \mathrm{min}$.

um free volume $\varphi_{\mathrm{e}}\left(T^{*}\right)$ of the melt. Above the Vogel temperature, however, the melt is still not completely frozen. There is a slight tendency that the volume $\varphi\left(T^{*}, \beta\right)$ will gradually relax towards its equilibrium value $\varphi_{\mathrm{e}}\left(T^{*}\right)$. During this process, the affinity increases from $a^{*}\left(T^{*}\right.$, $\beta)<0$ to $a^{*}=0$. Thus, if one anneals the frozen melt within the range $T_{\infty}<T^{*}<T_{\mathrm{g}}^{\prime}$, the free volume reaches a value $\varphi_{\mathrm{e}}\left(T^{*}\right) \leq \varphi(t) \leq \varphi\left(T^{*}, \beta\right)$ after the annealing time $t$. If one reheats the system at the same rate $|\beta|$, the hysteresis of the volume and the connected minimum value of the heat capacity become ever weaker with increasing annealing time $t$, the maximum value of the heat capacity, on the other hand, becomes ever larger (Figure 8). When the relaxing glass has finally reached the equilibrium value $\varphi_{\mathrm{e}}\left(T^{*}\right)\left(a^{*}=0\right)$, the hysteresis disappears completely upon heating. Since the undercooled melt is

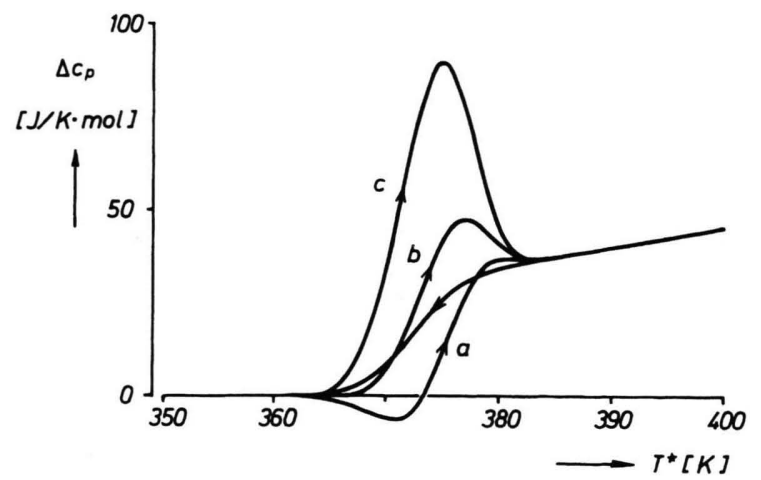

Fig. 8. Heat capacity $\Delta c_{p}$, according to (15b), when cooling down to $T^{*}=350 \mathrm{~K}$ at the rate $\beta=-1 \mathrm{~K} / \mathrm{min}$, "annealing" at $T^{*}=350 \mathrm{~K}$, and subsequently heating at the rate $\beta=+1 \mathrm{~K} / \mathrm{min}$. The initial values of the free volume $\varphi$ upon heating were a) $\varphi=0.0268907$ (the value which was attained upon cooling at $350 \mathrm{~K}$ ), b) $\varphi=0.0240000$, c) $\varphi=\varphi_{\mathrm{e}}=0.0193056$ (the equilibrium value of the melt at $350 \mathrm{~K}$ ).

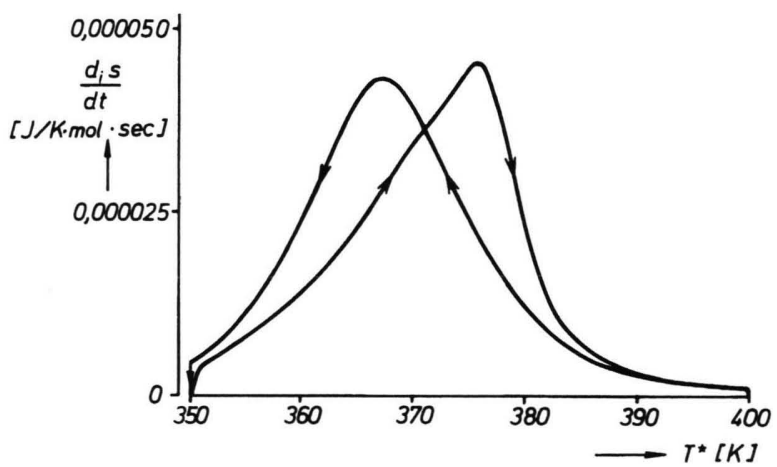

Fig. 9. Entropy production $\mathrm{d}_{i} s / \mathrm{d} t$, according to (50), when cooling down to $350 \mathrm{~K}$ at the rate $\beta=-1 \mathrm{~K} / \mathrm{min}$ and subsequently heating at the rate $\beta=+1 \mathrm{~K} / \mathrm{min}$. Although the free volume seems to be fully frozen below $365 \mathrm{~K}$ (see Fig. 3), there is still a considerable entropy production in this range. When cooling, the entropy production only disappears after reaching the Vogel temperature (at $340 \mathrm{~K}$ ). The position of the maxima of the entropy production indicates rather precisely the temperature at which the freezing of the free volume seems to be complete or alternatively the thawing of the free volume starts (compare Fig. 9 with Figure 3).

in a metastable state, it is possible that the free volume decreases even beyond the equilibrium value $\varphi_{\mathrm{e}}\left(T^{*}\right)$ upon annealing, and approaches the equilibrium value $\varphi_{\mathrm{e}}^{c}\left(T^{*}\right)<\varphi_{\mathrm{e}}\left(T^{*}\right)$ of the crystalline state. When judged with respect to the potential $g$ of the melt, the states $\varphi(t)<\varphi_{\mathrm{e}}\left(T^{*}\right)$ are non-equilibrium states with a positive affinity. If one heats up from such a state, the maximum of the heat capacity increases further. With $\varphi(t) \rightarrow 0$, we finally obtain $a^{*} \rightarrow \infty$ and $\Delta c_{p} \rightarrow \infty$. The increase in the maximum of the heat capacity with the annealing time, 
which is measured when heating a glassy frozen, annealed melt, has repeatedly led to the conclusion in the literature that the glass transition must be a masked phase transition in the sense of equilibrium thermodynamics [21]. But this is definitely not true. In these cases, the maximum of the heat capacity is clearly connected with the maximum of the affinity $a^{*} \neq 0$, which is characteristic for the non-equilibrium.

The entropy production $\mathrm{d}_{i} s / \mathrm{d} t$, according to (59), characteristic for the irreversibility of the processes, is represented in Fig. 9 for the case of the process Fig. 3 and Figure $7 \mathrm{~b}$. The entropy production disappears only at the beginning of the process at $T^{*}=T_{0}$ (internal equilibrium) and at the end of the process at $T^{*}=T_{\infty}$ (arrested equilibrium). In addition, it becomes very small if one switches from $\dot{T}^{*}=-\beta$ to $T^{*}=+\beta$ in the range $T_{\infty}<T^{*}<T_{\mathrm{g}}^{\prime}$ (in Fig. 9 at $T^{*}=350 \mathrm{~K}$ ). $\dot{T}$ passes through zero when switching (see further below), whereas $\dot{\zeta}$ is very small. The entropy production reaches a maximum value when cooling at the end of the freezing process and when heating at the beginning of the thawing process (compare Fig. 9 with Figure 3). The level of the maxima increases with $|\beta|$. Figure 9 shows that the glass transition generated at a finite rate $\dot{T}^{*}$ is an irreversible process in the whole interval $\left\langle T_{\infty}, T_{0}\right\rangle$. However, the contribution of the entropy production to the balance (47) of the entropy is relatively small during normal perturbation of the system. The maximum contribution of the entropy production to the balance is $0.95 \%$ with $\beta=-5 \mathrm{~K} / \mathrm{min}, 0.71 \%$ with $\beta=-1 \mathrm{~K} / \mathrm{min}$, and $0.55 \%$ with $\beta=-0.2 \mathrm{~K} / \mathrm{min}$.

According to (15), one can also write

$$
\dot{\zeta}=\frac{\Delta c_{p}}{T \sigma_{T p}} \dot{T} .
$$

Insertion of this expression into (49) leads to the interrelation

$$
\dot{s}=\frac{1}{T} c_{p} \dot{T},
$$

which completely corresponds to the equilibrium thermodynamics. However, the conclusion

$$
\dot{q}=c_{p} \dot{T}
$$

would be wrong, as $\dot{s}$ contains the entropy production which, according to Clausius, corresponds to a non-compensable heat. Instead of (66), (48) and (65) lead to the correct relation

$$
\dot{q}=\left(c_{p, \zeta}+\frac{\eta_{T p}}{T \sigma_{T p}} \Delta c_{p}\right) \dot{T} .
$$

(67) reduces to (66) only in the internal equilibrium (with $\eta_{T p} / T \sigma_{T p}=1$ ) and the arrested equilibrium (with $\Delta c_{p}=0$ ) [30]. However, $T \sigma_{T p}$ first deviates more strongly from $\eta_{T p}$ when $\Delta c_{p}$ approaches zero. Upon normal perturbation, therefore, the maximum deviation (66) from (67) remains below $1 \%$.

Finally, one should point out the following: $\dot{T}$ reacts spontaneously to a discontinuous change from $\dot{T}^{*}=-\left|\beta_{1}\right|$ to $\dot{T}^{*}=+\left|\beta_{2}\right|$ only in the arrested equilibrium. Above $T_{\infty}, T$ is subject to a retardation with respect to $T^{*}$ (Figure 5). $T$ then reacts to a discontinuous change of sign from $T^{*}<0$ to $T^{*}>0$ with a rapid, but still continuous change from $\dot{T}<0$ to $\dot{T}>0$. Therefore, $\dot{T}$ necessarily passes through zero during this transition. If $\dot{\zeta} \neq 0$ is valid, $\Delta c_{p}$, according to (15), passes through a singular infinity due to the sign reversal of $\dot{T}^{*}$. $\dot{\zeta} \approx 0$ holds if the sign reversal takes place at a temperature $T^{*}$ within the range $T_{\infty}<T^{*}<T_{\mathrm{g}}^{\prime}$. The temperature interval $\Delta T^{*}$, in which the singularity of $\Delta c_{p}$ becomes apparent, is then so small that the singularity can easily be overseen when solving the differential equations (52-54) with finite time steps $\Delta t$ (as in Figs. 7 and $8)$. In order to avoid the singularity, one must, in addition, postulate that with the sign reversal of the "flux" $\dot{T}^{*}$, the sign of the "force" $T^{*}-T$ also has to be changed. At the instant of the sign reversal of $\dot{T}^{*}, T$ must then be replaced by $2 T^{*}-T$. One should point out further that $\varphi$ can also be introduced as an internal variable in place of $\zeta=N_{1} / N_{2}$. With $L_{22}(0)=0.000000925 \mathrm{~mol} / \mathrm{J} \mathrm{sec}$ instead of $(64 \mathrm{c})$, one obtains practically the same results.

\section{Conclusions}

The thermodynamics of irreversible processes allows an, at least qualitative, understanding of the dynamics of the processes in the glass transition region on the basis of simple dynamic laws and a simple, certainly only approximately valid Gibbs fundamental equation. The glass transition generated with a finite cooling or heating rate is a dissipative process. Such processes require a clear differentiation between equilibrium and non-equilibrium quantities. In the thermodynamics of irreversible processes, this distinction is given a priori. The coupling coefficient $L_{22}$ and the affinity $a^{*}$ appear as determining quantities for the glass transition in the thermodynamics of irreversible processes. We associated the coupling coefficient with the fluidity of the melt according to Vogel, Fulcher, Tammann, and Hesse. Except for the sign, the affinity $a^{*}$ is essentially given by the partial free enthalpy of the vacancies in the melt. However, it still contains a certain correction which takes into account that in non-equilibrium the system temperature retards with respect to the temperature at which the heat exchange with the surroundings takes place. 
It becomes clear that the glass transition is a typical non-linear phenomenon. An explanation of the processes within the linear response theory, especially by the superposition of a spectrum of linear relaxation mechanisms, is, therefore, not very useful. Moreover, relaxation times prove to be auxiliary quantities, whose practical usefulness disappears when the differential equations describing the dynamics of the system are explicitly known. The Davies of Prigogine-Defay relations, which are often considered to be characteristic for the glass transition, do not have any significance in an irreversible process. These relations only become relevant with respect to the glass transition after a fictitious quasi-static cooling at the so-called Vogel temperature, which, according to our results, lies considerably below the temperature $T_{\mathrm{g}}$ usually designated as the glass temperature.

The fact that, according to our statements, the glass transition occurs during quasi-static cooling only at the Vogel temperature $T_{\infty}$ some ten degrees below $T_{\mathrm{g}}$, re-

[1] F. E. Simon, Erg. Exakt. Naturwiss. 9, 222 (1930).

[2] H. Breuer and G. Rehage, Kolloid-Z. u. Z. Polymere 216/217, 159 (1967).

[3] G. Rehage, J. Macromol. Sci.-Phys. B18, 423 (1980).

[4] I. Prigogine and R. Defay Traité de Thermodynamique, Tome I et II réunis, Desoer, Liège 1950.

[5] S. R. de Groot and P. Mazur Non-Equilibrium Thermodynamics, North-Holland Publishing Comp., Amsterdam 1962.

[6] H. Baur Einführung in die Thermodynamik der irreversiblen Prozesse, Wiss. Buchges., Darmstadt 1984.

[7] R. O. Davies Sur les soit-disant transitions de second ordre dans les milieux a relaxation, Comptes Rendus IUP, Société de Chimie Pysique, Paris 1952.

[8] R. O. Davies and G. O. Jones, Adv. Physics 2, 370 (1953).

[9] R. O. Davies and G. O. Jones, Proc. Roy. Soc. London A217, 26 (1953).

[10] N. Hirai and E. Eyring, J. Appl. Phys. 29, 810 (1958).

[11] N. Hirai and H. Eyring, J. Polymer Sci. 37, 51 (1959).

[12] H. Eyring and T. Ree, Proc. Natl. Acad. Sci. (US) 57, 526 (1961).

[13] J. I. Frenkel Kinetic Theory of Liquids, Oxford University Press, London 1946.

[14] P. J. Flory, J. Chem. Phys. 9, 660 (1941), 10, 51 (1942).

[15] E. A. Guggenheim, Thermodynamics, North-Holland Publishing Comp., Amsterdam 1959. minds us of the thesis of Gibbs et al. [31, 32], according to which the glass transition is supposed to occur as a second-order transition some ten degrees below $T_{\mathrm{g}}$ upon quasi-static cooling. As a matter of fact, the glass transition, which we find at $T_{\infty}$ with $T^{*}=\beta \rightarrow 0$, formally exhibits some characteristics of an Ehrenfest second-order transition. However, this is only true as long as we only consider a variation of the temperature. In the case of a pressure variation, the equilibrium value $\zeta_{\mathrm{e}}\left(T_{\infty}, p_{\infty}\right)$, which freezes at $T_{\infty}(p)$, becomes pressure-dependent. This fact alone proves that a freezing process can not be a second-order transition as defined by Ehrenfest [3]. Moreover, the quasi-static glass transition at the Vogel temperature $T_{\infty}$ appears as a limiting case with the dynamic quantities $\beta \rightarrow 0, L_{22} \rightarrow 0$, or $\eta \rightarrow \infty$ within the framework of the phenomenological thermodynamics of irreversible processes. In the pure equilibrium theory, dynamic quantities, such as $\beta, L_{22}, \eta$, or $a^{*}$ are completely irrelevant.

[16] G. Kanig, Kolloid-Z. u. Z. Polymere 233, 829 (1969)

[17] A. J. Kovacs, J. J. Aklonis, J. M. Hutchinson, and A. R. Ramos, J. Polymer Sci. Phys. Ed. 17, 1097 (1979).

[18] M. L. Williams, R. F. Landel, and J. D. Ferry, J. Amer. Chem. Soc. 77, 3701 (1955)

[19] T. G. Fox and P. J. Flory, J. Appl.-Phys. 21, 581 (1950).

[20] R. Simha and R. F. Boyer, J. Chem. Phys. 37, 1003 (1962).

[21] W. Wrasidlo, Adv. Polymer Sci. 13, 1 (1974).

[22] A. J. Kovacs, Adv. Polymer Sci. 3, 394 (1964).

[23] J. Jäckle, Rep. Prog. Phys. 49, 171 (1986).

[24] E.-J. Donth, Relaxation and Thermodynamics in Polymers, Akademie-Verlag, Berlin 1992.

[25] J. Meixner, Z. Physik 219, 79 (1969).

[26] I. Prigogine, Introduction to Thermodynamics of Irreversible Processes, Interscience Publishers, New York 1961.

[27] A. Q. Tool, J. Amer. Ceram. Soc. 29, 240 (1946).

[28] F. R. Schwarzl, Polymermechanik, Springer-Verlag, Berlin 1990.

[29] G. Strobl, The Physics of Polymers, Springer-Verlag, Berlin 1996.

[30] H. Baur and B. Wunderlich, J. Thermal Analysis, submitted.

[31] J. J. Gibbs and E. A. DiMarzio, J. Chem. Phys. 28, 373 (1958).

[32] G. Adam and J. H. Gibbs, J. Chem. Phys. 43, 139 (1965). 